\title{
El tratamiento térmico en rocas silíceas, un procedimiento técnico para la talla
}

\author{
The heat treatment of siliceous rocks, a technical process for knapping
}

Joana Boix Calbet (*)

\section{RESUMEN}

Se pretende mostrar un panorama general del estado actual de las investigaciones sobre el tratamiento térmico como procedimiento para la talla de rocas silíceas. Partiendo de varios autores, se ha desarrollado un programa experimental en el que se sintetizan los aspectos que se consideran más relevantes: las ventajas que supone el tratamiento térmico de la roca; los estigmas característicos que produce sobre ella, principalmente el cambio de color y el lustre térmico; y el momento de su aparición. Hemos experimentado con 24 muestras de un sílex nodular de color oscuro de Serra Llarga (Balaguer, Lleida) que no ha mostrado las mismas reacciones que otros tipos de sílex. Debemos destacar los resultados obtenidos en los pruebas de rugosidad de superficies, que han abierto una línea de investigación prometedora. Finalmente, apuntar la consideración del tratamiento térmico en un contexto técnico y económico más amplio, y no como un fin en sí mismo.

\begin{abstract}
This paper attempts to present on an overview of the use of heat treatment as a technical method for knapping siliceous rocks. We have developed an experimental programme in order to document the signs typical of thermal alteration (i.e. color change or thermal gloss) and the moment when these signs appear in the heating process and also to know the technical advantages resulting from heat treatment. We experimented with 24 samples of dark nodular flint from Serra Llarga (Balaguer, Lleida) that has not shown the same reactions as other types of flint. We have shown that surface roughness analysis is a new promising line of research for identifying thermal alteration in archaeological material. Finally, we should con-
\end{abstract}

(*) Becaria JaePredoc. Dpto. de Arqueología y Antropología. Institución Milá y Fontanals - Consejo Superior de Investigaciones Científicas (IMF-CSIC). C/ Egipcíaques 15. 08001 Barcelona. Correo e.: jboix@imf.csic.es

Recibido: 2-III-2011; aceptado: 15-IV-2011. sider the heat treatment in a more broadly technical and economic context, and not just as a method in itself.

Palabras clave: Arqueología experimental; Tecnología lítica; Alteraciones térmicas; Sílex; Prehistoria reciente; Europa Occidental.

Key words: Experimental Archaeology; Lithic technology; Thermal alterations; Flint; recent Prehistory; Western Europe.

\section{INTRODUCCIÓN}

El tratamiento térmico de las rocas silíceas para la mejora de su explotación mediante la talla no es una cuestión baladí. Representa un foco de interés sobre los procesos técnicos y el desarrollo tecnológico entre los grupos prehistóricos de especial relevancia en los estudios sobre el proceso de neolitización en Europa occidental. D. Crabtree y R. Butler (1964) publicaron el primer trabajo relacionado con esta temática. Desde entonces el corpus bibliográfico ha crecido de forma considerable centrándose en tres objetivos (Gibaja y Clemente 1997; Terradas y Gibaja 2001):

1. Qué ventajas supone la práctica de un tratamiento térmico.

2. Cuáles son los estigmas característicos resultantes.

3. En qué momento y cómo se manifiestan estos estigmas.

En general, el tratamiento térmico se ha venido considerando aislado y no dentro del proceso tecnológico más amplio de producción de instrumental lítico. Sin restar trascendencia a los cambios provocados por el calentamiento en la estructura, composición, orden interno, etc., de la 
materia prima nuestros intereses científicos no son los de la Química, la Física o la Geología sino el conocimiento de las sociedades humanas. En concreto aquí estudiamos los procedimientos técnicos desarrollados en el proceso de manufactura del instrumental lítico. Los cambios acaecidos en la roca tras un tratamiento térmico nos interesan en la medida en que nos aproximan a los objetivos especificados. Nos centramos en el desarrollo de criterios para el reconocimiento de los cambios documentados y de metodologías para interpretar su incidencia en la producción lítica. Nos interesa lo que hay detrás de un tratamiento térmico: qué materias primas se utilizan, en qué momento de la producción se practica, quién lo aplica, quién se beneficia de sus resultados y cuál es el uso final de los instrumentos obtenidos. El presente trabajo no soluciona estas cuestiones, pero constituye una parte previa y necesaria para determinar los rasgos discriminantes de un tratamiento térmico y sus objetivos. Primero tratamos qué es un tratamiento térmico y de qué criterios disponemos para reconocerlo. Segundo planteamos un programa experimental propio para observar, a distintas temperaturas, las alteraciones térmicas que sufre un sílex de tonalidades oscuras, independientemente de si se trata de nódulos o productos de talla. Finalmente replanteamos cuál debe ser la línea a seguir.

¿Pero a partir de cuándo y dónde podemos reconocer el tratamiento térmico? Algunos trabajos en curso en Sudáfrica, demostrarían el uso de la pirotecnología por parte de los primeros humanos modernos. La habrían utilizado para incrementar la calidad y la eficiencia de la silcreta en el yacimiento de Pinnacle Point 5-6 hace aproximadamente 164 mil años (ca.) (Brown et al. 2009) y para la talla a presión de preformas para la obtención de puntas bifaciales en el yacimiento de Bomblo Cave hace aproximadamente 75 mil años (ca.) (Mourre et al. 2010).

No obstante es en el Paleolítico Superior(1) y sobre todo durante el Neolítico cuando el tratamiento térmico parece haber tenido mayor importancia a juzgar por el número de casos y sus implicaciones técnicas. A finales del Neolítico esta práctica irá decayendo para desaparecer con

(1) F. Bordes (1969) publicó el primer caso de tratamiento térmico en un contexto arqueológico europeo sobre una hoja de laurel procedente de los estratos solutrenses del yacimiento francés de Laugerie-Haute. la generalización de la metalurgia, por lo menos en el Mediterráneo occidental.

El subcontinente indo-pakistaní es un caso excepcional, ya que el tratamiento térmico se documenta desde el inicio del Neolítico (Inizan y Lechevallier 1996) a la actualidad, utilizándose técnicas 'tradicionales' en procesos artesanales, vinculados con elementos ornamentales (Roux 2000). Posiblemente en el norte de América hay un mayor número de evidencias arqueológicas y documentación etnográfica con referencias de tratamiento térmico para la talla (Nagle 1914).

Etnográficamente se documenta prácticamente en todo el mundo: en las islas Andaman, en el Golfo de Bengala (Man 1883), Zimbaue (Robinson 1938), entre otras zonas. Desgraciadamente solo en el caso de la India, se informa sobre la importancia del tratamiento térmico en estas sociedades.

En la Península Ibérica, las primeras evidencias de tratamiento térmico corresponden a yacimientos de la zona de Levante y del suroeste del Paleolítico Superior: Cueva del Caldeirao en Portugal(2), Cueva de Ambrosio en Andalucía (Ripoll López et al. 1997) o Cueva del Parpalló en Gandía (Tiffagom 1998, 2006). Una mención especial merecen los yacimientos atribuidos al inicio del Neolítico del Sur de la Península Ibérica (Carvalho 2008). El estrecho nexo entre la producción laminar por presión y la aparición del tratamiento térmico quizás pueda relacionarse con influencias norteafricanas y acorde con su cronología proponerse un origen ibérico para el tratamiento térmico, asociado a la talla laminar, en el Neolítico de Europa occidental. Ejemplos de otras zonas no peninsulares son el sílex melado procedente del sureste francés (final del V milenio - inicios del IV milenio cal. BC), con el que se configuran preformas de núcleos cuya expansión geográfica abarca el Midi francés, Cataluña, Suiza y el Piemont-Ligurie (Binder 1998; Terradas y Gibaja 2001; Léa 2004, 2005); así como el tratamiento térmico sobre grandes láminas en contextos de Neolítico Final y Calcolítico en el centro de la Península Ibérica (Martín et al. 2009).

(2) Tiffagom (1998: 148) cita a Zilhão J. 1995: O Paleolitico superior da estremadura portuguesa. Tesis doctoral. Universidad de Lisboa, Facultad de Letras (inédita): 2 vols. 


\section{EL TRATAMIENTO TÉRMICO}

Tratamiento térmico, alteración térmica, annealing y pretratamiento térmico, son algunos de los conceptos que podemos encontrar en la bibliografía referente a la temática que aquí nos ocupa. Pero debemos ser cautos con su utilización. Terradas y Gibaja (2001) ya advierten del peligro de confundir el tratamiento térmico con las alteraciones térmicas, que en algunas publicaciones se utilizan como sinónimos.

En las ciencias de materiales el tratamiento térmico se define como un procedimiento técnico al que se someten algunos de ellos para mejorar sus propiedades mecánicas y físicas, básicamente la dureza, la resistencia y la tenacidad, mediante la aplicación de calor y el posterior enfriamiento (Kalpakjian y Schmid 2002).

Cuando se aplica a las rocas silíceas la mejora de las propiedades mecánicas se refleja en su forma de fractura. Crabtree y Butler (1964) ya demostraron en su momento a partir de réplicas experimentales que la aptitud(3) de muchas rocas silíceas para la talla mejora tras alterarlas térmicamente. Varios test mecánicos sobre el material experimental han demostrado que los cambios afectan la elasticidad de los materiales y su resistencia a la fractura. Dichos cambios tienen distintos efectos. La mejora más evidente es la necesidad de una menor fuerza del tallador en el momento de fracturar la roca (Crabtree y Butler 1964; Bleed y Meier 1980). Según Ahler (1983, cit. por Luedtke 1992: 100) dicha reducción en algún caso puede llegar al $50 \%$.

Se constata un mayor control en las operaciones relativas a la configuración de los bloques, la explotación de núcleos y el retoque de los soportes (Terradas y Gibaja 2001). El tratamiento térmico afecta a una de las propiedades mecánicas más importantes: la homogeneidad de sus propiedades. Su incremento hace la talla más predecible, reduce las posibilidades de fractura y la aparición de bordes reflejados (Luedtke 1992). Este mayor control resulta beneficioso sobre todo en zonas con períodos de inaccesibilidad a los recursos líticos debido a bajas temperaturas, nieve y

(3) Nos referimos a la mejora de las condiciones y propiedades que posibilitan la fractura intencionada de la roca mediante la aplicación controlada de una fuerza (presión, percusión) para la consecución de un objetivo preestablecido. heladas, que requieren un sistema que evite el derroche de la materia prima (Domanski y Weeb 2007). Destacamos que es en la zona sibero-mongola, durante el Paleolítico Superior, donde aparece el tratamiento térmico dirigido a la producción laminar (Inizan y Lechevallier 1996).

La alteración térmica permite la fabricación de productos con filos más agudos. Hay autores que han observado ángulos muchas veces inferiores a $\operatorname{los} 30^{\circ}$, ausentes en el mismo tipo de piezas sin alteración térmica (Rick 1978). La desventaja es que tiende a aumentar su fragilidad, haciendo depender sus posibilidades de utilización de la dureza de los materiales a manipular.

El tratamiento térmico es una ventaja en el momento de obtener productos de mayor longitud (Rick 1978; Bleed y Meier 1980). Mejora considerablemente la eficacia de la talla por presión y también la percusión mediante percutor blando y la percusión indirecta. El mayor control, facilidad y precisión en los procesos de transformación de la materia prima propicia su explotación mediante cualquier sistemática de talla.

Otra de las consecuencias del tratamiento térmico es un cambio en el aspecto externo de ciertas rocas silíceas hacia tonalidades más rojizas y superficies de aspecto más brillante. Ambos, según Domanski y Webb (2007), incrementarían el valor totémico o religioso del objeto por la simbología especial del color rojo en ciertas sociedades. Sin entrar en la simbología de ciertos colores, estos cambios visuales a nivel estético pueden bastar para practicar un tratamiento de este tipo. Por ejemplo las cuentas de cornalina de Cambay (India) reciben un tratamiento térmico sistemático para mejorar de la talla, pero sobre todo modificar su color (Roux 2000).

Estas mejoras/ventajas no se dan en todas las rocas silíceas de la misma manera. Principalmente son visibles en rocas microcristalinas cuyos cristales de cuarzo tienen menor tamaño: el sílex, el jaspe, el ágata, la calcedonia, la madera petrificada o la novacuolita. Una vez tratadas la fractura se asemeja a la de materiales como la obsidiana o el vidrio. Donde hay escasez de materias primas de buena calidad para la talla, parece que se ha utilizado el tratamiento térmico para mejorar la calidad de las existentes. Materiales macrocristalinos como la silcreta, la cuarcita o el cuarzo, también mejoran algo, pero no tanto 
como en las rocas microcristalinas. No obstante los resultados de las réplicas experimentales son muy variables. No todas las rocas, macrocristalinas o microcristalinas, con las que se ha experimentado han mostrado mejoras objetivas.

Como alteraciónes térmicas o termoalteraciones entendemos los cambios en la estructura y superficie de la roca tras someterla a una fuente de calor de forma premeditada o no. En cambio lo que define al tratamiento térmico es la intencionalidad y la planificación previa de acuerdo a unos objetivos prefijados.

Existe también el peligro de confundir tratamiento térmico y choque térmico. Esta alteración térmica se produce cuando la considerable diferencia de temperatura entre las partes de la roca, o entre la roca y su entorno fractura la roca (Luedtke 1992). Este choque térmico puede ser consecuencia de factores atmosféricos, de un contacto directo o fortuito con fuego o de un acto voluntario para fracturar el bloque. Al no buscar la modificación del bloque para la talla o para cambiar la coloración de la roca, no debería considerarse como tratamiento térmico en sentido estricto. Algunos autores han malinterpretado la documentación etnográfica viendo un tratamiento donde solo había un choque térmico. Este último punto puede ser motivo de discusión, porque ¿cuándo podemos considerar realmente que hay un tratamiento térmico? ¿También entraría el choque térmico destinado a extraer los bloques de sílex en algunos contextos mineros?

El éxito en un tratamiento térmico depende de unas condiciones determinadas de la materia prima, la temperatura y el tiempo. Pero aún controlando estas variables el tratamiento térmico no es un procedimiento preciso. Cada roca reacciona de manera distinta ante su exposición a los efectos de un foco de calor.

Hay un consenso en que el tratamiento térmico es un proceso largo y constante hasta llegar a la temperatura óptima, tras la cual debe haber un enfriamiento también progresivo y lento para evitar el choque térmico. Esta temperatura oscilaría entre los $250^{\circ} \mathrm{C}-350^{\circ} \mathrm{C}$ (Purdy y Brooks 1971), según las propiedades de la materia prima y el tamaño de la muestra. Por encima de esta temperatura la roca sufre daños que, en muchos casos, repercuten negativamente en la talla. Por debajo ningún cambio mejora significativamente sus propiedades. El tiempo de experimentación en horno de mufla puede variar entre $1 \mathrm{~h}$ y $72 \mathrm{~h}$. En estos casos la pieza se deja enfriar en su interior de manera que el descenso de la temperatura sea gradual. Basándonos en la información etnográfica, en un hogar al aire libre el tiempo de calentamiento sería de unas $24 \mathrm{~h}$ : en cada caso el calor sube lenta y progresivamente hasta llegar a la temperatura óptima y posteriormente se deja enfriar en el interior de la estructura de combustión utilizada hasta que se apaga la brasa.

La materia prima está infravalorada en la mayoría de los estudios, aunque a ningún autor se le escapa que cada litología da resultados diferentes. Otra variable a controlar es el tamaño del bloque. Crabtree y Butler (1964) constataron que el tratamiento térmico en los soportes comparativamente más delgados era mucho más exitoso. La observación, olvidada por muchos autores posteriores, ha sido recuperada recientemente por Mercieca y Hiscock (2008). En su trabajo experimentaron con cubos de silcreta de diferentes volúmenes para observar la respuesta térmica a distintas temperaturas. Constataron una clara interacción entre el volumen y la temperatura: las piezas con mayor volumen se fracturaron mucho antes que las de menor volumen. Esto influye también en el tratamiento simultáneo de distintas piezas, ya que para evitar que unas se fracturen antes que otras, todas tendrían que ser más o menos de un mismo tamaño o recibir un tratamiento individualizado.

En resumen, no podemos reducir el tratamiento térmico a la interacción tiempo y temperatura. Hace falta considerar también las propiedades específicas de la materia prima y su volumen.

¿Pero en qué momento del proceso de manufactura del instrumental lítico se tiene que realizar el tratamiento térmico? ¿Antes de la configuración del bloque de materia prima? ¿Antes de la obtención de los soportes? ¿Antes de dar forma a los soportes mediante el retoque? El tratamiento térmico no tiene un momento concreto de aplicación. Su elección depende de los objetivos buscados. Se puede aplicar en cualquier momento del proceso productivo dependiendo del desarrollo tecnológico, la capacidad de asumir el riesgo que comporta su aplicación y del objetivo final que se busca con el calentamiento de la roca. Por ejemplo en las cuentas de cornalina de la India el calentamiento se aplica en tres momentos distintos del proceso (Kenoyer et al. 1991). 


\section{CRITERIOS DE RECONOCIMIENTO DEL TRATAMIENTO TÉRMICO EN ROCAS SILÍCEAS}

Muchos trabajos experimentales se llevan a cabo para desarrollar métodos que permitan reconocer los efectos del tratamiento térmico en el material lítico. Desgraciadamente hasta el momento no hay ningún método concreto, ni macroscópico ni microscópico, para su identificación con total seguridad.

Parece claro que un tratamiento térmico no altera la mineralogía de la roca sino su estructura (Purdy y Brooks 1971) dado que la temperatura influye en la estructura de las rocas. Según Bertouille $(1989,1990)$, los cambios atmosféricos de temperatura acumulan tensiones en forma de dislocaciones e imperfecciones. Es el denominado 'fenómeno de fatiga'. Este altera la estructura de la roca, perjudicando sus propiedades para la ta1la. Para volver a modificar esta estructura necesitamos una energía similar o superior. La resultante de un tratamiento térmico puede producir una nueva redistribución de las dislocaciones, una restauración de la estructura y la reducción de los efectos de las tensiones. El fenómeno de fatiga se evita manteniendo el tratamiento en los límites de una temperatura óptima que modifique la estructura de la roca favoreciendo sus aptitudes para la talla.

El resultado del tratamiento térmico es también una mayor homogeneización de las propiedades texturales de la roca (Luedke 1992). Cuanto más diferente es el tamaño, forma y disposición de los cristales, peor es la talla. El tratamiento tiende a aumentar las propiedades isotrópicas de la roca, favoreciendo una mayor homogeneidad en su respuesta mecánica (Luedke 1992).

Aún hoy no hay una hipótesis única e inequívoca que explique los cambios internos de la roca al ser tratada térmicamente que modifican sus propiedades de talla. La primera hipótesis la propusieron D. Crabtree y Butler (1964: 2): Apparently, this what happens: Heat treatment causes recrystallization of the more coarsely fiberes and coarses microgranular silica minerals, which results in reduced crystal size a change in lustre from dull to greasy, an increase in elasticity of the material. Excessive heat, of course, will cause. A partir de esta se han propuesto otras, que no son excluyentes entre sí, al tratar la misma fenomenología desde distintas aproximaciones o fe- nómenos directamente vinculados entre sí. Luedke (1992) las agrupó en dos modelos: el silica fusion model (Purdy y Brooks 1971; Beauchamp y Purdy 1986; Griffiths et al. 1987), cuyas propuestas hacen referencia al relleno de los espacios intercristalinos y el crack model (Flenniken y Garrison 1975; Schindler et al. 1984) con las relativas a la creación de micro fracturas. El tercer modelo de 'recristalización' de Domanski y Weeb (2007) reúne las que postulan que los cristales se hacen más equigranulares. Todas estas teorías consideran la volatilización del agua, localizada en los poros y microfracturas del sílex, como principal agente en la mejora de las condiciones de talla de la roca (Griffiths et al. 1987; Domanski y Weeb 1992; Luedke 1992; McCutcheon y Kuehner 1997). Según las personas que tallan a nivel experimental esta reducción de la humedad favorecería el inicio de la microfracturación que se produce al aplicar una fuerza a un bloque o fragmento de sílex.

Para observar las alteraciones internas que se producen en la roca después de tratarla térmicamente se han utilizado analíticas como el Scanning Electron Microscope (SEM) o la termoluminiscencia. Por desgracia estas analíticas no son siempre asequibles por factores económicos o de disponibilidad de la infraestructura, aparte de suponer en ocasiones la destrucción total o parcial de la muestra. Por este motivo el tratamiento térmico sigue observándose controlando las alteraciones a nivel externo como el cambio en la coloración de la roca, la aparición del llamado lustre térmico y, ya cuando la temperatura resulta excesiva, de una serie de alteraciones más 'agresivas', perceptibles a simple vista. Sin embargo, debemos tener cuidado con estos cambios con un importante componente de subjetividad y difíciles de cuantificar.

El cambio de color, conocido como rubefacción (Fig. 1), aparece con temperaturas superiores a los $200^{\circ} \mathrm{C}$ en litologías cuyo contenido en partículas de hierro supera las 1100 ppm (Purdy y Brooks 1971). Según diversos autores el aumento de la temperatura oxida estas partículas. Al liberarse como consecuencia de la movilización del agua (Tixier 1984) aparece esta tonalidad más rojiza característica que varía según el grado de temperatura y la cantidad de impurezas.

El cambio de color no debe confundirse con la aparición de tonalidades blancas que experimentan todos los sílex cuando alcanzan una tem- 
peratura excesiva. Igualmente, otros tipos de pátinas, blancas o de otras tonalidades, pueden desarrollarse en el sílex como consecuencia de fenómenos naturales.

El lustre térmico es una de las primeras alteraciones térmicas que se evidencian en la roca. Se trata de un brillo identificable sólo en la superficie de los negativos de las extracciones realizadas después del tratamiento térmico (Fig. 1). La parte externa del bloque, núcleo o soporte que ha recibido tratamiento conserva un aspecto mate en comparación con ella. Además, las ondulaciones de la propagación del golpe quedan mejor impresas y de modo más regular (Terradas y Gibaja 2001). Este lustre aparece cuando las temperaturas llegan a los $200^{\circ} \mathrm{C}-300^{\circ} \mathrm{C}$, que es también la temperatura óptima según la mayoría de los autores para la mejora de las condiciones de talla. Esta temperatura depende de la materia prima, sin que exista un consenso acerca del origen de este brillo.

Las personas menos experimentadas pueden confundir el lustre térmico con otras alteraciones químicas y/o mecánicas como el pulido eólico que modifican la superficie de la pieza dejando características similares a las alteraciones térmicas.

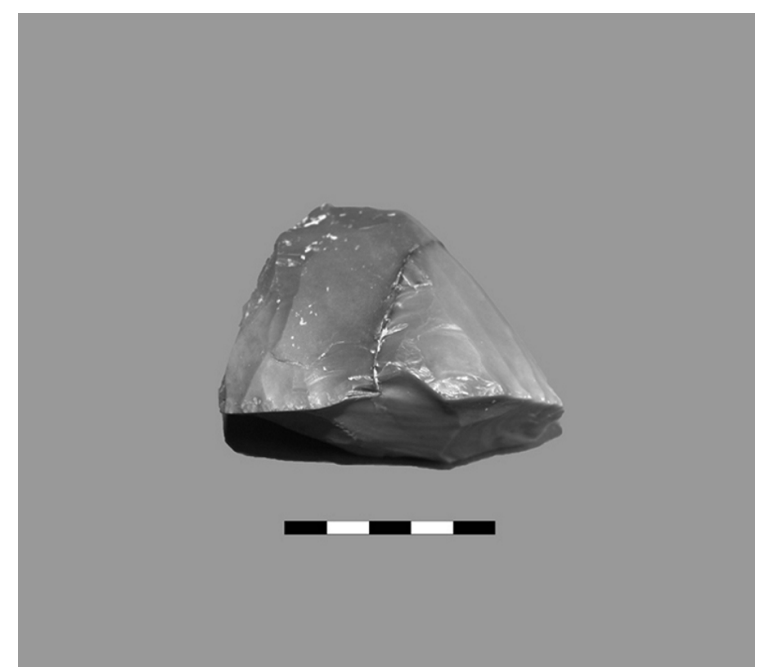

Fig. 1. Núcleo de sílex melado procedente de la estructura 70 del yacimiento de Can Gambús 1 (Sabadell, Barcelona), atribuible cronológicamente al Neolítico Medio, concretamente a la denominada Cultura de los Sepulcros de Fosa (finales del $\mathrm{V}$ a inicios del IV milenio cal BC) (lugar de depósito Museo de Historia de Sabadell) (Roig et al. 2010). En él se puede observar tanto la rubefacción de la superficie del núcleo, como el lustre térmico visible en las extracciones (fotografía J. Boix).
Se constatan otro tipo de alteraciones, mucho más agresivas e identificables con mayor facilidad, una vez se ha superado la horquilla en la que se sitúa la temperatura óptima. Pueden ser accidentales o consecuencia de una sobreexposición térmica/horaria durante la aplicación de un tratamiento térmico. Distintos tipos aparecen de forma gradual: cúpulas térmicas, fracturas, craquelado o escamado, pátina de color blanquecino o pardo, fragmentación en múltiples esquirlas e, incluso, desintegración completa (Clemente 1995, 1997; Paterson 1995) (Fig. 2).

Con frecuencia, el color o el lustre térmico no nos permiten identificar un tratamiento térmico con total seguridad, y en muchos casos sólo podremos constatar alteraciones debidas al contacto con alguna fuente de calor sin establecer su origen ni su intención. Así pues, resulta necesario buscar otros criterios de reconocimiento.

No hay una sola manera válida de determinar cuando un material está termoalterado de manera intencionada o fortuita. El criterio más diagnóstico es asociar los cambios de color y la aparición de lustre térmico en zonas concretas de la pieza con el estudio tecnológico y espacial de todo el conjunto lítico: su distribución espacial, la relación porcentual de piezas alteradas y no alteradas, la situación del tratamiento térmico en un momento concreto del proceso de transformación de las materias primas en productos, el nexo entre los objetivos que dirigen la producción y los restos derivados del proceso de talla, etc.

Otro punto a considerar debería ser la proximidad a fuentes de materia prima de yacimientos con evidencias de posible tratamiento térmico. No es un factor determinante, pero la necesidad de una gran cantidad de materia prima y el riesgo de accidentes inherentes a su tratamiento térmico aumentan las posibilidades de que este se lleve a cabo en las proximidades de los afloramientos explotados (Léa 2005). Esta circunstancia nos permite comparar el material termoalterado, del yacimiento estudiado con muestras de la misma materia prima no tratada térmicamente.

También cabría esperar de la fragilidad inherente a un conjunto lítico termoalterado una mayor tendencia a la fracturación. Debería ser posible observar un índice de fracturación más elevado en los productos alterados que en los que no lo están, siempre y cuando los productos tratados térmicamente hayan tenido un uso continuado. 


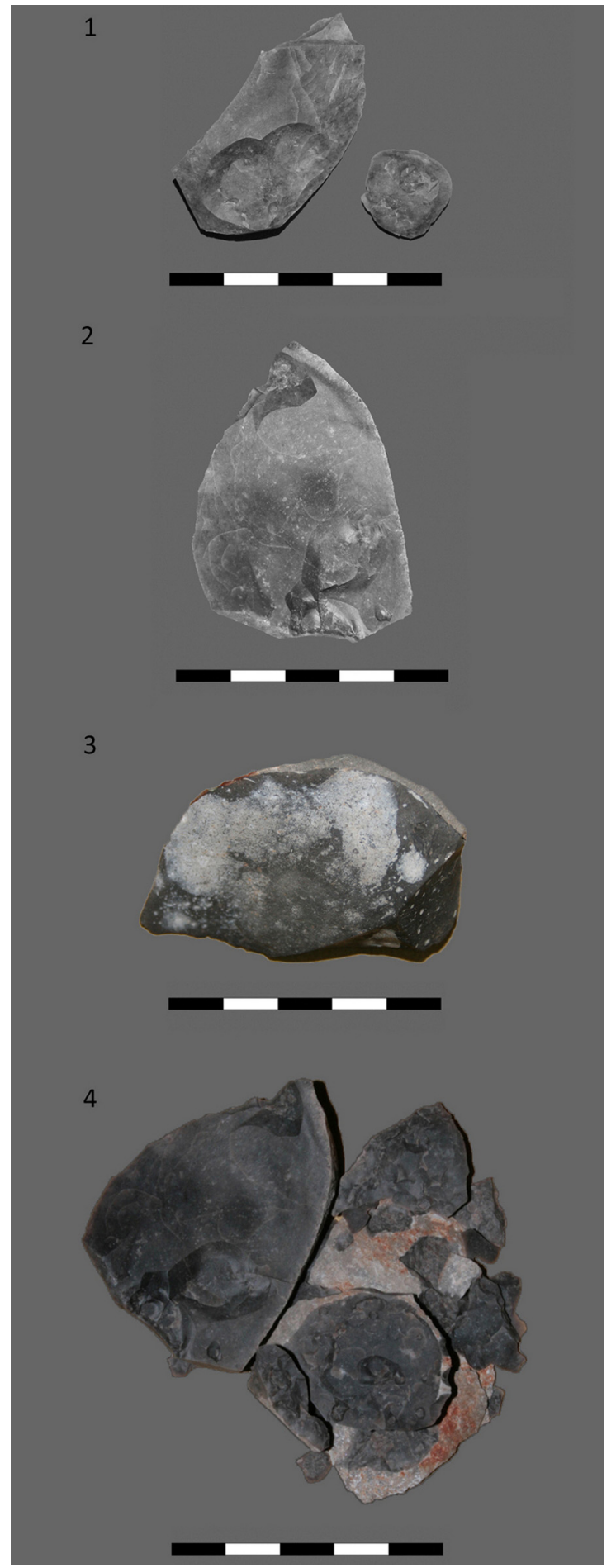

Fig. 2. Tratamiento térmico experimental de sílex de tonalidades oscuras, efectos de un tratamiento excesivo: 1. cúpulas térmicas, fragmento de la pieza B5.3; 2. escamado, pieza B7.2; 3. blanqueamiento, pieza B6.1 (no considerada en el experimento); 4. fragmentación en múltiples esquirlas, pieza B7.2 (fotografías J. Boix).
Otro criterio de reconocimiento de un tratamiento térmico serían las evidencias de su práctica in situ, a través de la identificación de las correspondientes estructuras de combustión (Fig. 3). Los ejemplos etnográficos sugieren una gran variabilidad de estrategias para la práctica de un tratamiento térmico de las rocas desde el hogar doméstico hasta aquel diseñado específicamente con la finalidad de calentar las rocas. Desgraciadamente esta gran variabilidad, las pequeñas dimensiones que pueden llegar a tener, la vinculación con otras evidencias antrópicas ajenas a la talla y/o la posibilidad de que estas estructuras estén aisladas dificultan su identificación.

En Zimbaue, los Naysalad depositaban los bloques en medio del hogar. Una vez calentados, sujetados contra una especie de yunque, se golpeaban con un martillo para extraer las lascas (Robinson 1938). En Norteamérica el grupo de los Plateau Shoshoni Indians colocaban las piezas en un hogar apagado y por encima hacían otro que mantenían durante 24 horas. En cambio los Reece River Shoshoni ponían las piezas bajo las cenizas durante 5 noches (Hester 1972, cit. por Gregg y Grybush 1976: 189). Los Kidja australianos, estudiados por Akerman (1979, cit. por Domanski y Webb 2007: 157), preparaban una cubeta de unos $50-60 \mathrm{~cm}$ de profundidad por $60 \mathrm{~cm}$ de diámetro. Antes de colocar el material encendían un fuego en el interior de la cubeta. Una vez apagado lo cubrían de arena y colocaban el material encima mezclado. Finalmente cubrían con tierra seca y lo dejaban aproximadamente 2 días. Mandeville y Flenikken (1974) describen un hogar destinado al tratamiento térmico donde, tras excavar la cubeta, primero se depositarían los materiales, se cubrirían de arena y encima se encendería un hogar.

La variabilidad de estructuras de combustión, aunque dificulta la rápida identificación de estos contextos arqueológicos, nos aporta mucha información sobre el grado de desarrollo de la producción del instrumental lítico tratado térmicamente. Desgraciadamente las evidencias arqueológicas son escasas, consecuencia posiblemente de la misma dinámica del tratamiento térmico donde para recuperar el material se destruiría la estructura. En la Península Ibérica tendríamos un posible ejemplo en una de las estructuras documentadas en el yacimiento del Campo de Fútbol de la Fábrica de Ladrillos Preresa (Getafe, Madrid) 

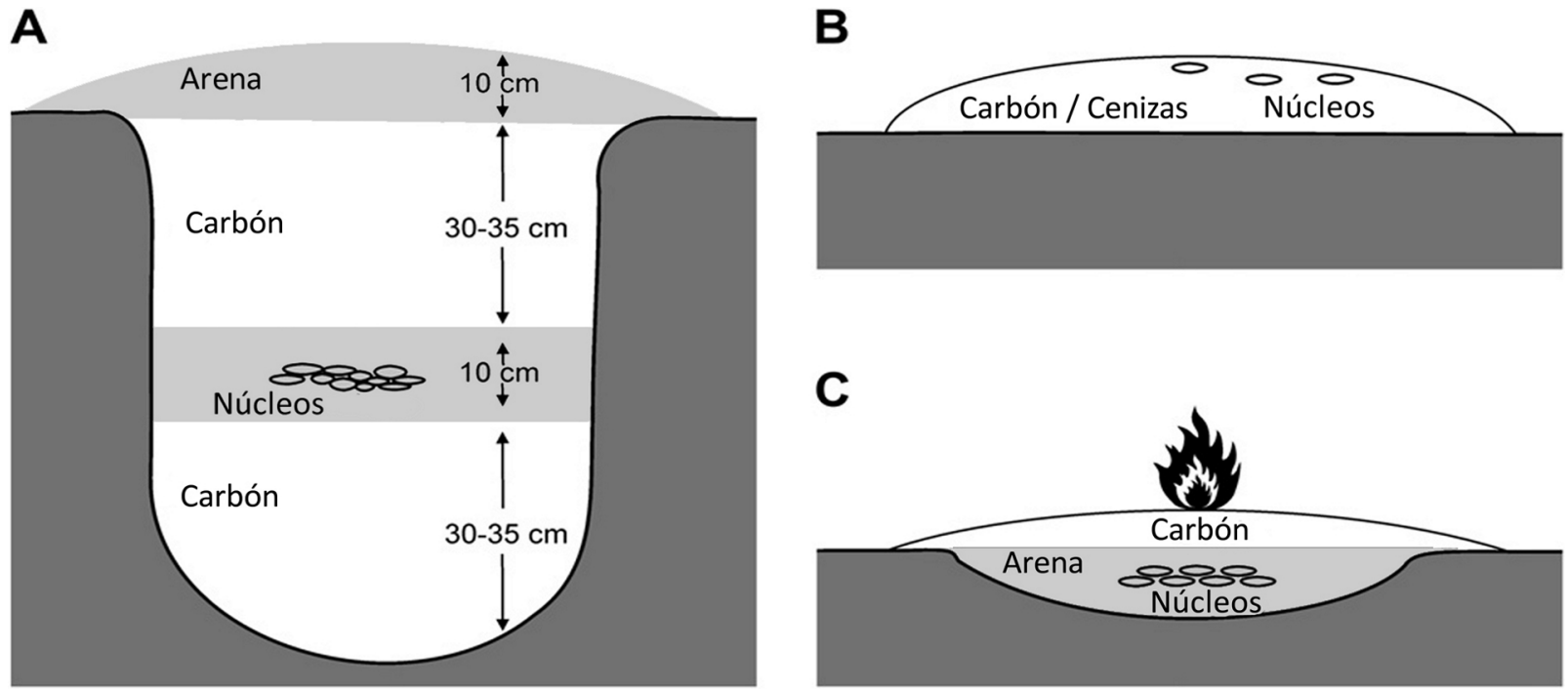

Fig. 3. Esquema de algunas de las posibles estructuras de combustión destinadas al tratamiento térmico (versión en castellano de Mercieca y Hiscock 2008: 2637, Fig. 3).

(Baena y Luque 1994). Se trata de una cubeta de aproximadamente $50 \mathrm{~cm}$ de profundidad y $50 \mathrm{~cm}$ de diámetro donde se intercalarían niveles de arena, limpia y con carbón, y donde mayoritariamente se han recuperado restos líticos.

\section{UN EJEMPLO EXPERIMENTAL: EL TRATAMIENTO TÉRMICO EN EL SÍLEX NODULAR DE SERRA LLARGA (BALAGUER, LLEIDA)}

Las dificultades para afirmar la existencia de un tratamiento térmico y su finalidad hacen necesaria la experimentación en laboratorio bajo condiciones favorables para el control de las variables consideradas más relevantes de los materiales alterados, así como también entender los procesos de cambio en la estructura de la roca. Para este primer experimento, hemos utilizado un sílex nodular encajado en un cuerpo de calizas lacustres, de facies micrítica, adscritas a la Formación de Castell (Serra Llarga, Balaguer, Lleida) (Fig. 4). Esta formación tiene una edad priaboniense terminal y forma parte del sistema lacustre de La Noguera que, a su vez, se incluye en una de las secuencias deposicionales del relleno sedimentario del sector oriental de la cuenca del Ebro (Anadón et al. 1989). La morfología de los nódulos de sílex es esférica o plana con una longi- tud que oscila entre los 5 y los 20 o $25 \mathrm{~cm}$. El sílex es de color negro-gris (tabla Munsell: 10YR 4/1, dark grey; 7.5R 3/0, very grey dark; $2.5 \mathrm{YR}$ $3 / 0$, very grey dark.), opaco y de grano fino. Sus superficies pueden presentar alteraciones, principalmente fracturas y pátinas de desilicificación.

Seleccionamos este tipo de sílex por tres motivos: su explotación contrastada a lo largo de la Prehistoria, la posibilidad de que hubiera sido objeto de un tratamiento térmico y su coloración oscura que permitía adivinar una respuesta distinta a la de otras litologías explotadas en otros contextos arqueológicos. A partir de la talla por percusión directa de los nódulos de un tamaño entre 10 y $15 \mathrm{~cm}$ seleccionamos 24 lascas de dimensiones similares (Tab. 1). Muestra suficiente para observar el comportamiento de este sílex de características diferentes a las de otros sometidos a experimentación.

El sílex se ha calentado en un horno de mufla que puede alcanzar hasta $1.000^{\circ} \mathrm{C}$. Su controlador de rampas permite programar subidas de temperatura a tiempo determinado en una sola hornada lo que evita llegar a la temperatura final demasiado rápido y permite mayor control del tiempo de exposición del material al calentamiento.

Las piezas se han colocado en el interior de recipientes cerámicos llenos de arena. Al evitar que el calor les llegue de forma súbita se excluye un choque térmico y que estallen. Utilizamos 


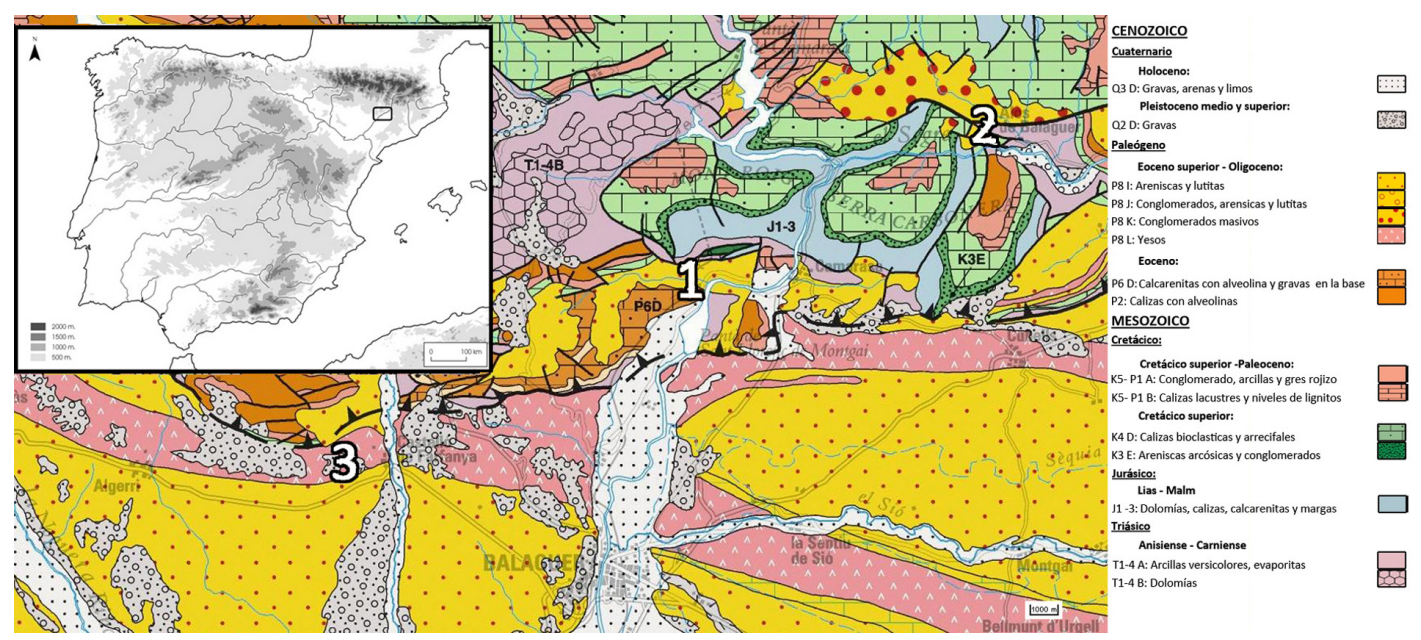

Fig. 4. Serra Llarga (Balaguer, Lleida), zona de adquisición del sílex utilizado en la experimentación e indicios de su explotación arqueológica: 1. La Roca dels Bous (Paleolítico Medio); 2. La Cova del Parco (Paleolítico Superior a Bronce Final); 3. Auvelles (Epipaleolítico a Bronce Medio). Mapa Geológico 1:250.000 (2002, propiedad del Instituto Cartográfico de Cataluña, disponible en www.icc.cat; fecha de consulta on-line 18-XI-2011).

\begin{tabular}{|c|c|c|c|c|c|c|}
\hline \multirow{2}{*}{ Pieza } & \multirow{2}{*}{${ }^{\circ} \mathrm{C}$} & \multirow{2}{*}{ Calentamiento } & \multirow{2}{*}{ Enfriamiento } & \multicolumn{2}{|c|}{ Peso } & \multirow{2}{*}{ Volumen } \\
\hline & & & & previo & posterior & \\
\hline B1.1 & 150 & $1 \mathrm{~h} 30^{\prime}$ & Lento (20 h) & $42,63 \mathrm{~g}$ & $42,55 \mathrm{~g}$ & $48 \times 70 \times 14 \mathrm{~mm}$ \\
\hline B3.2 & 150 & $1 \mathrm{~h} 30^{\prime}$ & Lento $(20 \mathrm{~h})$ & $27,19 \mathrm{~g}$ & $27,07 \mathrm{~g}$ & $42 \times 39 \times 22 \mathrm{~mm}$ \\
\hline B1.9 & 150 & $1 \mathrm{~h} 30^{\prime}$ & Rápido (exterior) & $31,37 \mathrm{~g}$ & $31,29 \mathrm{~g}$ & $45 \times 44 \times 20 \mathrm{~mm}$ \\
\hline B4.3 & 150 & $1 \mathrm{~h} 30^{\prime}$ & Rápido (exterior) & $33,54 \mathrm{~g}$ & $33,36 \mathrm{~g}$ & $47 \times 40 \times 20 \mathrm{~mm}$ \\
\hline B1.3 & 200 & $2 \mathrm{~h}$ & Lento $(20 \mathrm{~h})$ & $24,91 \mathrm{~g}$ & $24,76 \mathrm{~g}$ & $32 \times 64 \times 13 \mathrm{~mm}$ \\
\hline B2.4 & 200 & $2 \mathrm{~h}$ & Lento $(20 \mathrm{~h})$ & $28,04 \mathrm{~g}$ & $27,81 \mathrm{~g}$ & $40 \times 33 \times 23 \mathrm{~mm}$ \\
\hline B3.3 & 200 & $2 \mathrm{~h}$ & Rápido (exterior) & $24,91 \mathrm{~g}$ & $24,74 \mathrm{~g}$ & $55 \times 22 \times 25 \mathrm{~mm}$ \\
\hline B3.5 & 200 & $2 \mathrm{~h}$ & Rápido (exterior) & $26,38 \mathrm{~g}$ & $26,33 \mathrm{~g}$ & $53 \times 36 \times 14 \mathrm{~mm}$ \\
\hline B2.3 & 250 & 2 h 30 & Lento $(20 \mathrm{~h})$ & $18,42 \mathrm{~g}$ & $18,20 \mathrm{~g}$ & $52 \times 20 \times 21 \mathrm{~mm}$ \\
\hline B3.8 & 250 & 2 h $30^{\prime}$ & Lento $(20 \mathrm{~h})$ & $20,63 \mathrm{~g}$ & $20,61 \mathrm{~g}$ & $47 \times 48 \times 9 \mathrm{~mm}$ \\
\hline B2.2 & 250 & 2 h $30^{\prime}$ & Rápido (exterior) & $25,92 \mathrm{~g}$ & $25,75 \mathrm{~g}$ & $36 \times 52 \times 15 \mathrm{~mm}$ \\
\hline B5.4 & 250 & 2 h $30^{\prime}$ & Rápido (exterior) & $29,99 \mathrm{~g}$ & $29,78 \mathrm{~g}$ & $36 \times 72 \times 15 \mathrm{~mm}$ \\
\hline B3.6 & 300 & $3 \mathrm{~h}$ & Lento (20 h) & $19,35 \mathrm{~g}$ & $19,18 \mathrm{~g}$ & $44 \times 49 \times 11 \mathrm{~mm}$ \\
\hline B4.2 & 300 & $3 \mathrm{~h}$ & Lento $(20 \mathrm{~h})$ & $36.37 \mathrm{~g}$ & $36,32 \mathrm{~g}$ & $40 \times 49 \times 24 \mathrm{~mm}$ \\
\hline B4.5 & 300 & $3 \mathrm{~h}$ & Rápido (exterior) & $22,64 \mathrm{~g}$ & $22,61 \mathrm{~g}$ & $56 \times 34 \times 15 \mathrm{~mm}$ \\
\hline B1.10 & 300 & $3 \mathrm{~h}$ & Rápido (exterior) & $64,41 \mathrm{~g}$ & $64,13 \mathrm{~g}$ & $46 \times 51 \times 27 \mathrm{~mm}$ \\
\hline B5.5 & 350 & 3 h $30^{\prime}$ & Lento (20 h) & $43,06 \mathrm{~g}$ & $42,59 \mathrm{~g}$ & $71 \times 38 \times 18 \mathrm{~mm}$ \\
\hline B4.1 & 350 & 3 h $30^{\prime}$ & Lento (20 h) & $21,65 \mathrm{~g}$ & $21,58 \mathrm{~g}$ & $55 \times 31 \times 16 \mathrm{~mm}$ \\
\hline B5.6 & 350 & 3 h $30^{\prime}$ & Rápido (exterior) & $56,40 \mathrm{~g}$ & $56,79 \mathrm{~g}$ & $64 \times 51 \times 18 \mathrm{~mm}$ \\
\hline B3.4 & 350 & 3 h $30^{\prime}$ & Rápido (exterior) & $31,59 \mathrm{~g}$ & $31,32 \mathrm{~g}$ & $62 \times 32 \times 16 \mathrm{~mm}$ \\
\hline B7.2 & 400 & $4 \mathrm{~h}$ & Lento $(20 \mathrm{~h})$ & $37,94 \mathrm{~g}$ & $37,17 \mathrm{~g}$ & $53 \times 41 \times 15 \mathrm{~mm}$ \\
\hline B5.3 & 400 & $4 \mathrm{~h}$ & Lento (20 h) & $26,30 \mathrm{~g}$ & - & $63 \times 40 \times 13 \mathrm{~mm}$ \\
\hline B2.1 & 400 & $4 \mathrm{~h}$ & Rápido (exterior) & $71,62 \mathrm{~g}$ & $70,58 \mathrm{~g}$ & $62 \times 42 \times 25 \mathrm{~mm}$ \\
\hline B1.2 & 400 & $4 \mathrm{~h}$ & Rápido (exterior) & $33,35 \mathrm{~g}$ & $33,23 \mathrm{~g}$ & $67 \times 52 \times 8 \mathrm{~mm}$ \\
\hline B.1.Rug. & 350 & 3 h 30 , & Lento $(20 \mathrm{~h})$ & - & - & - \\
\hline B.5.Rug. & 350 & 3 h $30^{\prime}$ & Lento $(20 \mathrm{~h})$ & - & - & - \\
\hline
\end{tabular}

Tab. 1. Medidas de control tomadas a las piezas experimentales antes y después de ser calentadas en un horno de mufla. Las últimas dos piezas corresponden a las muestras utilizadas exclusivamente para el test de rugosidad. 
arena como protector ya que es un buen conductor térmico, que asegura una transmisión regular del calor favoreciendo un calentamiento y enfriamiento homogéneos y graduales. Además, es un material fácil de conseguir y existen referencias etnográficas de su uso para dicha finalidad.

Las variables que la mayoría de los autores apuntan como importantes son la temperatura, el sílex se ha calentado $150^{\circ} \mathrm{C}, 200^{\circ} \mathrm{C}, 250^{\circ} \mathrm{C}$, $300^{\circ} \mathrm{C}, 350{ }^{\circ} \mathrm{C}$ y $400^{\circ} \mathrm{C}$; el tiempo de calentamiento, con un aumento progresivo de $50^{\circ} \mathrm{C}$ cada 30 minutos y el de enfriamiento, lento, aproximadamente 20 horas, más rápido con la extracción inmediata de la pieza y a temperatura ambiente. Además se controlan el peso y el volumen.

Se ha medido el color, el volumen y el peso de cada pieza en las distintas fases de la experimentación. Las piezas se depositan de dos en dos en los recipientes cerámicos rellenos de arena. Estos se introducen en la mufla también de dos en dos calentándose hasta la temperatura deseada en cada momento. Una vez alcanzada se apaga la mufla y se saca al exterior solo uno de los recipientes, así conseguimos enfriar rápidamente dos piezas a temperatura ambiente y dos lentamente. Finalmente se practican extracciones a las piezas para observar si se ha producido el lustre térmico esperado.

Cada tipo de roca tiene reacciones diferentes frente a un tratamiento térmico y todas ellas son útiles para ampliar nuestro conocimiento. Pero debemos puntualizar que el sílex de Serra Llarga (Balaguer, Lleida) no es una materia agradecida para la observación de las reacciones de una roca silícea expuesta a una fuente controlada de calor. Como presumíamos, este sílex no ha mostrado las mismas reacciones aparecidas en otras rocas empleadas en experimentaciones realizadas por otros investigadores.

El cambio en la coloración por exposición a un foco de calor, en este caso, al tratarse de un sílex oscuro y con un bajo contenido de partículas férricas, no se ha producido. Recordar que, según Purdy y Brooks (1971), los sílex con más alto contenido de partículas férricas, más de 1.100 ppm, son los únicos que cambian el color como consecuencia del proceso de oxidación de las mismas cuando se llega a temperaturas próximas a $\operatorname{los} 200^{\circ} \mathrm{C}$.

El lustre térmico, la alteración que muchos autores destacan como la más relevante para la identificación del tratamiento térmico, aparece alrededor de $\operatorname{los} 300^{\circ} \mathrm{C}$. Pero, la diferencia esperable entre las partes lustrosas y sin lustre no es tan contrastada como por ejemplo en el sílex provenzal (Terradas y Gibaja 2001). En los sílex calentados a $350^{\circ} \mathrm{C}$ y enfriados de manera rápida este lustre térmico no se da. ¿La aparición del lustre térmico está entonces más relacionada con el tiempo y tipo de enfriamiento que con la temperatura considerada óptima? Los futuros experimentos tendrán que incidir en esta cuestión. Si el lustre es uno de los puntos claves para reconocer el tratamiento térmico, ¿cómo lo identificamos en estos casos? ¿Será un tratamiento más común de lo que creemos?

En el caso que nos ocupa, la temperatura óptima de calentamiento se sitúa entre los 300 y los $400^{\circ} \mathrm{C}$, y es cuando aparecen las primeras evidencias de lustre térmico en un momento previo a la aparición de los daños. A partir de $\operatorname{los} 400^{\circ} \mathrm{C}$ la roca sufre daños considerables, se fractura. $\mathrm{Su}$ aparición gradual es también mucho más rápida de lo esperable. Los daños no siguen tampoco ningún patrón fijo.

Como indicamos, el tamaño es una de las variables a tener en cuenta al practicar un tratamiento térmico: a mayor tamaño más propensión al choque térmico debido a la mayor diferencia de temperatura entre el exterior y el interior. Este es el caso de la pieza $\mathrm{B} 1.2$, que a $400{ }^{\circ} \mathrm{C}$ solo ha desarrollado alguna cúpula térmica, todo lo contrario de las otras calentadas a la misma temperatura y ligeramente más gruesas, que han quedado totalmente fragmentadas.

La pérdida de peso relacionada con la pérdida interna del agua localizada en los poros y microfracturas de la roca, es insignificante. Por eso no creemos que sea una variable relevante.

Tras el tratamiento térmico, a simple vista y también con el tacto, se observan diferencias entre las nuevas superficies generadas a partir de las extracciones y las no modificadas, que permiten constatar el lustre térmico. Creemos necesario aplicar algún test que confirme si esta diferencia existe realmente $\mathrm{y}$, en caso afirmativo, buscar alguna manera de objetivarla y cuantificarla.

Para intentar medir la rugosidad a diversos aumentos, y cuantificarla mediante índices establecidos a partir de su representación tridimensional, hemos recurrido al Stereo Explorer 2.2. Este programa informático nos permite estas mediciones y un control directo sobre el microscopio, en nuestro caso, uno estereoscópico Leica 

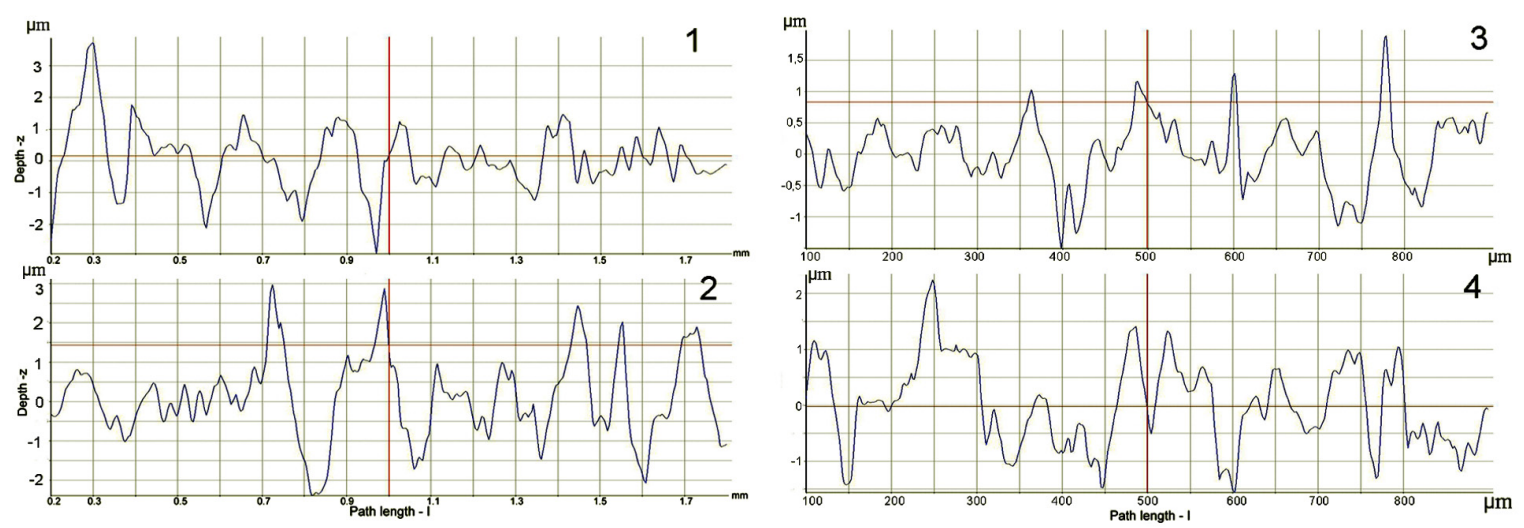

Fig. 5. Tratamiento térmico experimental de sílex oscuro (Serra Llarga, Balaguer, Lleida). Representación gráfica de las mediciones, Mean roughness profile, observadas en la superficie de la pieza B.1.Rugosidad a 15 aumentos $(1,3) \mathrm{y}$ 30 aumentos $(2,4)$ antes $(1,3)$ y después $(2,4)$ del calentamiento controlado en mufla.

\begin{tabular}{|c|c|c|c|c|c|}
\hline AUMENTOS & $\begin{array}{c}\text { ZONA } \\
\text { MEDICIÓN }\end{array}$ & $\begin{array}{c}\text { ANTES DE } \\
\text { CALENTAR }\end{array}$ & $\begin{array}{c}\text { DESPUÉS DE } \\
\text { CALENTAR }\end{array}$ & $\begin{array}{c}\text { SOBRE LAS } \\
\text { EXTRACCIONES } \\
\text { SIN LUSTRE }\end{array}$ & $\begin{array}{c}\text { SOBRE LAS } \\
\text { EXTRACCIONES } \\
\text { CON LUSTRE }\end{array}$ \\
\hline $15 \mathrm{X}$ & 1 & $2,609 \mu \mathrm{m}$ & $2,528 \mu \mathrm{m}$ & $4,444 \mu \mathrm{m}$ & $4,892 \mu \mathrm{m}$ \\
$15 \mathrm{X}$ & 2 & $2,06 \mu \mathrm{m}$ & $2,516 \mu \mathrm{m}$ & $4,759 \mu \mathrm{m}$ & $5,454 \mu \mathrm{m}$ \\
$30 \mathrm{X}$ & 1 & $1,345 \mu \mathrm{m}$ & $2,065 \mu \mathrm{m}$ & $3,001 \mu \mathrm{m}$ & $3,09 \mu \mathrm{m}$ \\
$30 \mathrm{X}$ & 2 & $1,615 \mu \mathrm{m}$ & $1,818 \mu \mathrm{m}$ & $2,693 \mu \mathrm{m}$ & $3,493 \mu \mathrm{m}$ \\
\hline
\end{tabular}

Tab. 2. Valores RA - Mean average roughness of profiles- de las medidas tomadas a 15 y 30 aumentos sobre la pieza B.1.Rug antes y después de calentarla y sobre las extracciones realizadas tras el calentamiento. Significativo es el cambio que se produce entre las medidas tomadas antes del calentamiento y aquellas tomadas sobre las extracciones, independientemente del grado de lustre que presenten.

MZ16FA con foco motorizado. Dicho control se ejerce sobre las cámaras, los aumentos y las profundidades en las superficies, permitiendo crear modelos 3D, así como también analizar perfiles, áreas y volúmenes.

El programa de medidas proporciona gran cantidad de parámetros. Nos hemos fijado en los que hacen referencia directa a la rugosidad $y$, en especial, en el parámetro Ra (Mean average roughness of profiles) o valor promedio de rugosidad en $\mu \mathrm{m}(4)$.

En esta primera prueba medimos la rugosidad de una roca antes y después del tratamiento térmico, y sobre las extracciones practicadas a pos-

(4) El mismo programa nos advierte que esta medida puede resultar un tanto 'errónea' ya que nos da una media general. teriori. Las tomamos a 15 y 30 aumentos y a 45 y 60 aumentos. Finalmente desestimamos los últimos resultados debido a que, al incrementar los aumentos, se reduce correlativamente la zona a medir y por tanto crece la dificultad de evidenciar y comparar las diferencias. En cambio, a 15 y 30 aumentos, sí obtenemos diferencias significativas al comparar la superficie antes y después de las extracciones (Fig. 5), estas prácticamente se duplican (Tab. 2). Esto corroboraría la hipótesis de partida según la cual las superficies de aspecto mate y más lustroso que aparecen en las extracciones posteriores a un tratamiento térmico son apreciablemente distintas, siendo estas últimas las de mayor índice de rugosidad. Podemos objetivar tales diferencias de rugosidad, pero debemos profundizar aún más en su significado. Nos falta ampliar la muestra y realizar más estadísti- 
cas que nos ayuden a entender mejor lo que sucede, pero se nos ha abierto una línea de investigación prometedora.

\section{COMENTARIOS FINALES}

For archaeologists human responses to heat treatment are more significant than the physical changes which take place in heat stones and, thus, should be the major focus of the study (Bleed y Meier 1980: 506). Esta frase resume la principal problemática a resolver. En todos los ámbitos de la investigación arqueológica nos centramos con demasiada frecuencia en adaptar técnicas desarrolladas por otras disciplinas, intentando buscar su 'aplicabilidad' arqueológica. Solemos priorizar la novedad de la técnica frente a su capacidad de resolución de problemáticas arqueológicas, substituyendo nuestro verdadero objeto de conocimiento por nuestro objeto de estudio.

Uno de los peligros más habituales cuando experimentamos en arqueología es confundir medios y fines. Por ejemplo, en los trabajos sobre tratamiento térmico, tenemos una idea bastante clara de las reacciones de las rocas frente al calor, pero nos falta identificar la importancia de este tipo de tratamiento en los procesos de producción.

Es evidente que se deben seguir investigando los cambios estructurales internos que sufre la roca al ser alterada térmicamente, pero nuestro trabajo como arqueólogos está en aprovechar los datos de todos estos cambios internos que sean relevantes para nuestra investigación. Es evidente que el estudio de los cambios físicos nos aporta datos indispensables. Pero los cambios estructurales tienen que considerarse secundarios. En la medida de lo posible tendríamos que trabajar más estrechamente con equipos/personas formados en otras disciplinas, intentando desarrollar una investigación de carácter marcadamente interdisciplinar.

Debemos empezar a poner mucho más énfasis en otro tipo de criterio diagnóstico como el propio contexto arqueológico. La clave para identificar un tratamiento térmico no está en las alteraciones resultantes, sino en la relación que se establece entre ellas, la interpretación global de todo el conjunto lítico y la distribución espacial de este conjunto en el seno del yacimiento, en relación al resto de productos y actividades.
Los programas experimentales sobre el tratamiento térmico, al igual que sobre otros aspectos de la tecnología lítica, deberían desarrollarse en un contexto técnico y económico más amplio. En definitiva, ese tratamiento no es más que una opción técnica, un segmento más del proceso de producción del instrumental lítico. Su aplicación depende de unas necesidades sociales concretas $\mathrm{y}$ de la oferta medioambiental del territorio en que opera esta sociedad. El tratamiento térmico responde a la voluntad de conseguir una materia prima con unas propiedades nuevas, diferentes a las originales y destinadas a solventar unas necesidades igualmente diferentes.

Este trabajo ha sido una primera incursión al tratamiento térmico en rocas silíceas como procedimiento técnico para la talla. Hemos visto qué cambios sustanciales experimenta el sílex, cómo se manifiestan a nivel superficial y sus posibilidades interpretativas en el seno de un estudio sobre la producción técnica en la Prehistoria. Igualmente, se adivinan líneas potenciales de desarrollo de esta investigación en el futuro, entre las cuales el estudio de la rugosidad de las superficies termoalteradas parece ser muy prometedora. Esperemos que una correcta identificación de las distintas alteraciones térmicas y su correcta contextualización permitan interpretar adecuadamente los aportes que supone el tratamiento térmico como procedimiento técnico en la manufactura de instrumentos líticos y su significación tecnológica.

Valorando en su conjunto la experimentación realizada y formulada en este trabajo, pensamos que no puede darse por terminada. Al hacerlo han aparecido más interrogantes que respuestas. Es un proceso natural y lógico en la formulación de una investigación experimental cuyo objetivo es contrastar determinadas hipótesis. Estos interrogantes plantean a la vez nuevos retos científicos y posibilitan detectar los puntos débiles del programa experimental. Gracias a ello esperamos replantearlo con el objetivo de resolver las deficiencias y carencias. El logro de estos objetivos otorgará una mayor solidez a los resultados obtenidos.

\section{AGRADECIMIENTOS}

Esta experimentación se realizó como trabajo final de tercer ciclo titulado El tractament tèrmic 
en roques silícies com a procediment tècnic per a la talla: estat de la qüestió, presentado en el Dpto. de Prehistoria de la Universidad Autónoma de Barcelona. Agradezco a Xavier Terradas, Institución Milá y Fontanals - Consejo Superior de Investigaciones Científicas (IMF-CSIC), mi director de tesis, a Juan Francisco Gibaja (IMFCSIC) e Ignacio Clemente (IMF-CSIC), así como a los correctores anónimos, sus consejos y correcciones durante la redacción del artículo.

\section{BIBLIOGRAFÍA}

Ahler, S. A. 1983: "Heat treatment of Knife River Flint". Lithic Technology 12: 1-8.

Akerman, K. 1979: "Heat and lithic Technology in the Kimberleys, W.A.". Archaeology and Physical Anthropology in Oceania 14: 144-151.

Anadón, P.; Cabrera, L.; Colldeforns, B. y Sáez, A. 1989: "Los sistemas del Eoceno superior y Oligoceno del sector oriental de la Cueva del Ebro". Acta Geologica Hispanica 24 (3-4): 205-230.

Baena, J. y Luque, M. 1994: "La industria lítica". En C. Blasco (ed.): El horizonte campaniforme de la región de Madrid en el centenario de Ciempozuelos. Universidad Autónoma de Madrid. Madrid: 173-223.

Beauchamp, E. K. y Purdy, B. A. 1986: "Decrease in fracture toughness of chert by heat treatment". Journal of Materials Science 21: 1963-1966.

Bertouille, H. 1989: Théories physiques et mathématiques de la taille des outils préhistoriques. Cahiers du quaternaire 15. Bordeux.

Bertouille, H. 1990: "Restauration de la structure des roches par traitement thermique". En M. R. Séroinie-Vivien y M. Lenoir (eds.): Le silex de sa genèse à l'outil. Cahiers du Quaternaire 17 (I). Bordeaux: 167-169.

Binder, D. 1998: "Silex blond et complexité des assemblages lithiques dans le Néolithique liguroprovençal". En A. D'Anna y D. Binder (eds.): Production et identité culturelle. Actualité de la recherché. Éditions APDCA. Antibes: 111-128.

Bleed, P. y Meier, M. 1980: "An objective test of the effects of heat treatment of flakeable stone". American Antiquity 45 (3): 502-507.

Bordes, F. 1969: "Traitement thermique du silex au Solutréen". Bulletin de la Société Préhistorique Française 66 (7): 197.

Brown, K. S.; Marean, C. W.; Herries, A. I. R.; Jacobs, Z.; Tribolo, Ch.; Braun, D.; Roberts, D. L.; Meyer, M. C. y Bernatchez, J. 2009: "Fire As an Engineering Tool of Early Modern Humans". Science 325: 859-862.
Carvalho, A. F. 2008: A Neolitizaçao do Portugal Meridional. Os exemplos do Maciço Calcário Estremenho e do Algarve Occidental. Promontoria Monográfica 12. Universidade do Algarve. Faro.

Clemente-Conte, I. 1995: "Sílex y lustre térmico en el Paleolítico medio ¿Alteración o técnica de talla? El ejemplo de Mediona I (Alt Penedès, Barcelona)". 1. Congresso de Arqueologia Peninsular (Porto, 12-18 de octubre de 1993). Trabalhos de Antropologia e Etnologia XXXV (3): 37-47.

Clemente-Conte, I. 1997: "Thermal alterations of flint implements and the conservation of microwear polish: preliminary experimental observations". En A. Ramos Millán y M. A. Bustillo (eds.): Siliceous rocks and culture. Universidad de Granada. Granada: 525-535.

Crabtree, D. y Butler, R. 1964: "Notes on experiments in flintknapping. 1: heat treatment of silica materials". Tebiwa 7 (1): 1-6.

Domanski, M. y Webb, J. A. 1992: "Effect of heat treatment on siliceous rocks used in prehistoric lithic technology". Journal of Archaeological Science 19: 601-614.

Domanski, M. y Webb, J. A. 2007: “A review of heat treatment research". Lithic Technolgy 32 (2): 153194.

Flenniken, J. J. y Garrison, E. G. 1975: “Termally altered novacuolite and stone tool manufacturing techniques". Journal of Field Archaeology 12 (1/2): 125-131.

Gibaja, J. F. y Clemente, I. 1997: “El tratamiento térmico del sílex y sus repercusiones en la determinación de los rastros de uso. Algunos ejemplos del neolítico en Cataluña". Revista d'arqueologia de Ponent 7: 153-160.

Gregg, M. L. y Grybush, R. J. 1976: "Thermally altered siliceous stone from prehistoric contexts: intentional versus unintentional alteration". American Antiquity 41 (2): 189-192.

Griffiths, D. R.; Bergman, C. A.; Clayton, C. J.; Ohnuma, K.; Robins, G. V. y Seeley, N. J. 1987: "Experimental investigation of the heat treatment of flint". En G. Sieveking y M. H. Newcomer (eds.): The Human Uses of Flint and Chert. Cambridge University Press. Cambridge: 43-52.

Hester, T. R. 1972: "Ethnographic evidence for the thermal alteration of siliceous stone". Tebiwa 12: 63-65.

Inizan, M. L. y Lechevallier, M. 1996: "Deux traditions techniques de la pierre taillée dans le souscotinent Indo-Pakistanais: le débitage par pression et le traitement thermique". Páleorient 22 (1): 145152.

Kalpakjian, S. y Schmid, S. R. 2002: Manufactura, ingeniería y tecnología. Pearson Educación. Méjico.

Kenoyer, J. M.; Vidale, M. y Kumar Bhan, K. 1991: "Contemporary Stone Beadmaking in Khambhat, 
India: Patterns of Craft Specialization and Organization of Production as Reflected in the Archaeological Record". World Archaeology 23 (1): 44-63.

Léa, V. 2004: "Centres de production et diffusion des silex béouliens au Chasséen". Gallia Préhistoire 46: 231-250.

Léa, V. 2005: "Raw, pre-heated or ready to use: discovering specialist supply systems for flint industries in mid-Neolithic (Chassey culture) communities in southern France". Antiquity 79: 51-65.

Luedtke, B. E. 1992: Archaeologist's guide to chert and flint. University of California. Los Angeles.

Man, E. H. 1883: "On the aboriginal inhabitants of the Andaman Islands (Part III)". The Journal of Anthropological Intitute of Great Britain and Ireland 12: 327-434.

Mandeville, M. D. y Flenniken, J. J. 1974: “A comparison of the flaking qualities of Nehawka chert before and after thermal pretreatment". Plains Anthropologist 19: 146-148.

Martín, P.; Rubio, D.; Cuartero, F.; Baena, J. y Fabián, F. 2009: "La talla laminar por presión en el centro Peninsular". En J. F. Gibaja, X. Terradas, T. Palomo y X. Clop (eds.): Les grans fulles de sílex. Europa al final de la Prehistòria. Actes. Monografies 13. Museu d'Arqueologia de Catalunya. Barcelona: 47-54.

McCutcheon, P. T. y Kuehner, S. M. 1997: "From macroscopic to microscopic: understanding prehistoric heat treatment of stone tools". En A. Ramos Millán y M. A. Bustillo (eds.): Siliceous rocks and culture. Universidad de Granada. Granada: 447-462.

Mercieca, A. y Hiscock, P. 2008: "Experimentals insights into alternative strategies of lithic heat treatment". Journal of Archaerological Science 35: 2634-2639.

Mourre, V.; Villa, P. y Henshilwood, Ch. S. 2010: "Early Use of Pressure Flaking on Lithic Artifacts at Blombos Cave, South Africa". Science 330: 659-662.

Nagle, E. 1914: "Arrow-chipping by means of fire and water". American Anthropologist 16: 140.

Paterson, L. W. 1995: "Thermal damage of chert". Lithic Technology 20 (1): 72-80.

Purdy, B. A. y Brooks H. K. 1971: "Thermal alteration of silica minerals: an archaeological approach". Science, 173 (3994): 322-325.
Rick, J. W. 1978: Heat Altered Cherts of the Lower Illinois Valley: An Experimental Study in Prehistoric Technology. Northwestern University Archaeological Program, Prehistoric Records No. 2. Evanston, Illinois.

Ripoll López, S.; Pérez Marin, S.; López Moreno de Redrojo, J. R.; Martos Romero, J. A. y Muniz Pérez, M. 1997: "Las estructuras de combustionen la cueva de Ambrosio. Estudio preliminar". En J. M. Fullola y N. Soler (eds.): El Mon mediterrani des prés del Pleniglacial (18000-12000 BP). Museu d'Arqueologia de Catalunya, Série monogràfica 17. Girona: 399-409.

Robinson, T. R. 1938: “A survival of flake-technique in Southern Rhodesia". Man 38: 208.

Roig, J.; Coll, J. M.; Gibaja, J. F.; Chambon, P.; Villar, V.; Ruiz, J.; Terradas, X. y Subirà, M. E. 2010: "La necrópolis de Can Gambús-1 (Sabadell, Barcelona). Nuevos conocimientos sobre las prácticas funerarias durante el neolítico medio en el noreste de la Península Ibérica". Trabajos de Prehistoria, 67 (1): 59-84.

Roux, V. 2000: Cornaline de l'Inde. Des practiques techniques de Cambay aux techno-systèmes de l'Indus. Éditions de la Maison des Sciences de l'homme. Paris.

Schindler, D. L; Hatch, J. W; Hay, C. A. y Bradt R. C. 1984: "Thermal alteration of Bald Eagle Jasper: Authors' reply to Patterson". American Antiquity 49 (1): 173-177.

Terradas, X. y Gibaja, J. F. 2001: “El tratamiento térmico en la producción lítica: el ejemplo del neolítico medio catalán”. Cypsela 13: 31-58.

Tiffagom, M. 1998: “Témoignages d'un traitement thermique des feuilles de laurier dans le Solutréen Supérieur de la Grotte du Parpalló (Gandia, Espagne)". Paleo 10: 147-161.

Tiffagom, M. 2006: De la Pierre a l'Homme. Essai sur une paléoanthropologie soloutréenne. Eraul 113. Liege.

Tixier, J. 1984: “Le debitage par pression". En J. Tixier (ed.): Préhistoire de la pierre taillée, 2: économie du débitage laminaire: technologie et expérimentation: IIIe table ronde de technologie lithique, MeudonBellevue, 1982. Cercle de recherche et d'Études Préhistoriques. Paris: 57-70. 